\title{
ON $\mathscr{M}$-HARMONIC BLOCH SPACE
}

\author{
MIROLJUB JEVTIĆ AND MIROSLAV PAVLOVIĆ
}

(Communicated by Clifford J. Earle)

\begin{abstract}
We show that many of the characterizations of analytic Bloch functions also characterize $\mathscr{M}$-harmonic Bloch functions.
\end{abstract}

\section{INTRODUCTION}

The class of analytic Bloch functions on the unit disc and the unit ball $B$ in $C^{n}$ is well known, and it has been studied by many authors ([3], [4], [5], [6], [8], [13], [14]). In this note $\mathscr{M}$-harmonic Bloch functions on $B$ are studied. Our results show that many of the characterizations of analytic Bloch functions also characterize $\mathscr{M}$-harmonic Bloch functions. Some other characterizations of $\mathscr{M}$-harmonic Bloch functions are given in [9].

To state our main result we need some notation. As in [12], we say that a function $u \in C^{2}(B)$ is $\mathscr{M}$-harmonic in $B, u \in \mathscr{M}$, if $\widetilde{\Delta} u(z)=0$ for every $z \in$ $B$. The operator $\widetilde{\Delta}$ is the invariant Laplacian defined by $\widetilde{\Delta} u(z)=\Delta\left(u \circ \varphi_{z}\right)(0)$, $z \in B$, where $\Delta$ is the ordinary Laplacian and $\varphi_{z}$ is the standard automorphism of $B$ taking 0 to $z$ (see [12]).

For $f \in C^{1}(B), D f=\left(\partial f / \partial z_{1}, \ldots, \partial f / \partial z_{n}\right)$ denotes the complex gradient of $f$, and $\nabla f=\left(\partial f / \partial x_{1}, \ldots, \partial f / \partial x_{2 n}\right), z_{k}=x_{2 k-1}+i x_{2 k}, k=$ $1,2, \ldots, n$, denotes the real gradient of $f$.

For $f \in C^{1}(B)$ let $\widetilde{D} f(z)=D\left(f \circ \varphi_{z}\right)(0), z \in B$, and $\tilde{\nabla} f(z)=\nabla\left(f \circ \varphi_{z}\right)(0)$, $z \in B$, be the invariant complex gradient of $f$ and the invariant real gradient of $f$, respectively.

If $f \in C^{1}(B)$ let

$$
\left|\nabla_{T} f(z)\right|^{2}=2\left(|D f(z)|^{2}-|R f(z)|^{2}+|D \bar{f}(z)|^{2}-|R \bar{f}(z)|^{2}\right), \quad z \in B,
$$

be the tangential gradient of $f$. As usual, $R$ denotes the radial derivative $R=\sum_{j=1}^{n} z_{j} \partial / \partial z_{j}$.

We say that $f \in \mathscr{M}$ is $\mathscr{M}$-harmonic Bloch function, $f \in \mathscr{M} \mathscr{B}$, if $\|f\|_{\mathscr{B}}=$ $\sup _{z \in B}|\widetilde{\nabla} f(z)|<\infty$.

We define the little $\mathscr{M}$-harmonic Bloch space $\mathscr{M} \mathscr{B}_{0}$ to be the subspace of $\mathscr{M} \mathscr{B}$ for which $\lim _{|z| \rightarrow 1}|\tilde{\nabla} f(z)|=0$.

Received by the editors November 14, 1991 and, in revised form, June 10, 1993.

1991 Mathematics Subject Classification. Primary 32A37, 30D45.

$K e y$ words and phrases. Bloch space, $\mathscr{M}$-harmonic functions. 
Theorem 1. Let $f \in \mathscr{M}$. Then the following are equivalent:

(1) $f$ is a $\mathscr{M}$-harmonic Bloch function,

(2) $\sup _{z \in B}\left(\widetilde{\Delta}|f|^{2}\right)^{1 / 2}<\infty$,

(3) $\sup _{z \in B}\left(1-|z|^{2}\right)^{1 / 2}\left|\nabla_{T} f(z)\right|<\infty$,

(4) $\sup _{z \in B}\left(1-|z|^{2}\right)|\nabla f(z)|<\infty$,

(5) $\sup _{z \in B}\left(1-|z|^{2}\right)(|R f(z)|+|\bar{R} f(z)|)<\infty$, where $\bar{R}=\sum_{j=1}^{n} \overline{z_{j}} \partial / \partial \overline{z_{j}}$.

In [14] Theorem 1 was proved for analytic functions. The proof, based on the Cauchy integral formula, shows that, if $f: B \mapsto C$ is analytic and $|\nabla f(z)|$ grows at most as fast as $1 /\left(1-|z|^{2}\right)$, then the directional derivatives of $f$ in directions perpendicular to the radial directions grow at most as fast as $1 /\left(1-|z|^{2}\right)^{1 / 2}$. Using the integral representation formulas for derivatives of $\mathscr{M}$-harmonic functions obtained in [1] we show that $\mathscr{M}$-harmonic functions also behave twice as well in the complex-tangential directions.

The equivalences of Theorem 1 carry over to the little $\mathscr{M}$-harmonic Bloch space as is shown in the following theorem.

Theorem 2. Let $f \in \mathscr{M}$. Then the following statements are equivalent:

(1) $f \in \mathscr{M} \mathscr{B}_{0}$,

(2) $\left(\widetilde{\Delta}|f|^{2}(z)\right)^{1 / 2}=o(1),|z| \rightarrow 1$,

(3) $\left|\nabla_{T} f(z)\right|=o(1 / \sqrt{1-|z|}),|z| \rightarrow 1$,

(4) $|\nabla f(z)|=o(1 /(1-|z|)),|z| \rightarrow 1$,

(5) $\left(1-|z|^{2}\right)(|R f(z)|+|R \bar{f}(z)|)=o(1),|z| \ldots$ :

We omit details.

For $f \in \mathscr{M}$ let

$$
\partial f(z)=\left(\frac{\partial f}{\partial z_{1}}(z), \ldots, \frac{\partial f}{\partial z_{n}}(z), \frac{\partial f}{\partial \bar{z}_{1}}(z), \ldots, \frac{\partial f}{\partial \bar{z}_{n}}(z)\right)
$$

and for any positive integer $m$ we write $\partial^{m} f(z)=\left(\partial^{\alpha} \bar{\partial}^{\beta} f(z)\right)_{|\alpha|+|\beta|=m}$ and $\left|\partial^{m} f(z)\right|^{2}=\sum_{|\alpha|+|\beta|=m}\left|\partial^{\alpha} \bar{\partial}^{\beta} f(z)\right|^{2}$, where

$$
\partial^{\alpha} \cdot \bar{\partial}^{\beta} f(z)=\frac{\partial^{|\alpha|+|\beta|} f(z)}{\partial z_{1}^{\alpha_{1}}, \ldots, \partial z_{n}^{\alpha_{n}} \partial{\overline{z_{1}}}^{\beta_{1}}, \ldots, \partial{\overline{z_{n}}}^{\beta_{n}}},
$$

$\alpha$ and $\beta$ are multi-indices.

Our second result is the following theorem which relates the Bloch norm of an $\mathscr{M}$-harmonic function with quantities involving integrals of the higher-order derivative of the function. Even though $\|f\|_{\mathscr{B}}, f \in \mathscr{M}$, is not a norm, we refer to $\|f\|_{\mathscr{B}}$ as the Bloch norm of the function $f$. The quantity $|f(0)|+\|f\|_{\mathscr{B}}$ defines a norm on the linear space $\mathscr{M}$ which, equipped with this norm, is a Banach space.

Theorem 3. Let $0<p<\infty, 0<r<1$, and $m \in N$. Then for a $\mathscr{M}$-harmonic function $f$ the following quantities are equivalent:

(i) $\|f\|_{\mathscr{B}}<\infty$,

(ii) $\sup _{z \in B}(1-|z|)|\partial f(z)|<\infty$,

(iii) $\sup _{z \in B}(1-|z|)^{m}\left|\partial^{m} f(z)\right|<\infty$,

(iv) $\sup _{z \in B} \int_{E_{r}(z)}\left|\partial^{m} f(w)\right|^{p}(1-|w|)^{m p-n-1} d v(w)<\infty$.

For analytic functions Theorem 3 was proved in [6], [13]. 


\section{Proof of Theorem 1}

For $a \in B$ and $0<r<1$ let $E_{r}(a)=\left\{z \in B:\left|\varphi_{a}(z)\right|<r\right\}$. The measure $\tau$ defined on $B$ by $d \tau(z)=\left(1-|z|^{2}\right)^{-n-1} d \nu(z)$, where $\nu$ denotes the $2 n$-dimensional Lebesgue measure on $B$ normalized so that $\nu(B)=1$, is $\mathscr{M}$-invariant (see [12]). In particular, $\tau\left(E_{r}(a)\right)=\tau(r B), a \in B, 0<r<1$. Any unexplained notation is as in [12].

Lemma 2.1. Let $0<r<1$. There is a constant $C$ such that if $f \in \mathscr{M}$, then

(a) $\left|T_{i j} R f(w)\right| \leq C\left(1-|w|^{2}\right)^{-1 / 2} \int_{E_{r}(w)}|R f(z)| d \tau(z), w \in B$,

(b) $\left|T_{i j} \bar{R} f(w)\right| \leq C\left(1-|w|^{2}\right)^{-1 / 2} \int_{E_{r}(w)}|\bar{R} f(z)| d \tau(z), w \in B$. As usual, $T_{i j}=\overline{z_{i}} \partial / \partial z_{j}-\overline{z_{j}} \partial / \partial z_{i}$ are tangential derivatives.

Here and elsewhere constants are denoted by $C$ which may indicate a different constant from one occurrence to the next.

Proof. (a) By the formula (1.3) in [1]

$$
R f(w)=\int_{S} \frac{R f\left(\varphi_{w}(\rho \xi)\right)}{1-\langle\rho \xi, w\rangle} d \sigma(\xi), \quad w \in B, 0<\rho<1 .
$$

Multiplying this equality by $2 n \rho^{2 n-1}\left(1-\rho^{2}\right)^{-n-1} h(\rho) d \rho$, where $h$ is a radial function which belongs to $C^{\infty}(B)$ with compact support in $B$ such that $\int_{B} h(z) d \tau(z)=1$, and then integrating from 0 to 1 and using the invariance of the measure $\tau$, we get

$$
\begin{aligned}
R f(w) & =\int_{B} h\left(\varphi_{w}(z)\right) \frac{1}{1-\left\langle\varphi_{w}(z), w\right\rangle} R f(z) d \tau(z) \\
& =\int_{B} h\left(\varphi_{z}(w)\right) \frac{1-\langle z, w\rangle}{1-|w|^{2}} R f(z) d \tau(z),
\end{aligned}
$$

by Theorem 2.2 .5 ([12], p. 28).

Denote the components of $\varphi_{z}$ by $\varphi_{1}(\cdot, z), \ldots, \varphi_{n}(\cdot, z)$. Since these are holomorphic in $B$ with $\sup _{z, w \in B}\left|\varphi_{m}(z, w)\right|=1,1 \leq m \leq n$, we have $\left|T_{i j} \varphi_{m}(w, z)\right| \leq C\left(1-|w|^{2}\right)^{-1 / 2}$, by Lemma 2.3 in [2] (see also [10]).

Note that $T_{i j}(1-\langle z, w\rangle) /\left(1-|w|^{2}\right)=0$ (here the operator $T_{i j}$ denotes differentiation with respect to $w)$.

Now the chain rule gives

$$
\begin{aligned}
\left|T_{i j} R f(w)\right| & =\mid \int_{B} h^{\prime}\left(\varphi_{z}(w)\right)\left[\sum_{m=1}^{n} \frac{\overline{\varphi_{m}}(w, z)}{2\left|\varphi_{z}(w)\right|} T_{i j} \varphi_{m}(w, z)\right] \\
& \cdot \frac{1-\langle z, w\rangle}{1-|w|^{2}} R f(z) d \tau(z) \mid \\
\leq & C\left(1-|w|^{2}\right)^{-1 / 2} \int_{B}\left|h^{\prime}\left(\varphi_{z}(w)\right)\right| \frac{|1-\langle z, w\rangle|}{1-|w|^{2}}|R f(z)| d \tau(z) .
\end{aligned}
$$

By a suitable choice of a function $h$ we obtain

$$
\left|T_{i j} R f(w)\right| \leq C\left(1-|w|^{2}\right)^{-1 / 2} \int_{E_{r}(w)}|R f(z)| d \tau(z), \quad \text { for some } 0<r<1 .
$$

Here, we have used the fact that $|1-\langle z, w\rangle| \cong 1-|w|^{2}$, if $z \in E_{r}(w)$. 

we get

(b) Since $\bar{f} \in \mathscr{M}$ and $\bar{R} f=\overline{R \bar{f}}$, from the formula for $R f$, obtained above,

$$
\bar{R} f(w)=\int_{B} h\left(\varphi_{z}(w)\right) \frac{1-\langle w, z\rangle}{1-|w|^{2}} \bar{R} f(z) d \tau(z)
$$

and consequently

$$
\begin{aligned}
\left|T_{i j} \bar{R} f(w)\right| \leq & \int_{B}\left|h^{\prime}\left(\varphi_{w}(z)\right)\right|\left|\sum_{m=1}^{n} \frac{\overline{\varphi_{m}}(w, z)}{2\left|\varphi_{z}(w)\right|} T_{i j} \varphi_{m}(w, z)\right| \\
& \quad \frac{|1-\langle w, z\rangle|}{1-|w|^{2}}|\bar{R} f(z)| d \tau(z) \\
& +\int_{B}\left|h\left(\varphi_{w}(z)\right)\right|\left|T_{i j}(1-\langle w, z\rangle)\right| \frac{|\bar{R} f(z)|}{1-|w|^{2}} d \tau(z)=I_{1}+I_{2} .
\end{aligned}
$$

Note that here we have used the fact that

$$
T_{i j}\left(\frac{1-\langle w, z\rangle}{1-|w|^{2}}\right)=\frac{1}{1-|w|^{2}} T_{i j}(1-\langle w, z\rangle) .
$$

If the operator $T_{i j}$ denotes differentiation with respect to $w$ as above, and $z \in E_{r}(w)$ is written as $z=\varphi_{w}(u)$ (with $u \in r B$ ), then it is easily seen that

$$
\left|T_{i j}(1-\langle w, z\rangle)\right|=\left|\frac{S_{w}\left(u_{i} w_{j}-u_{j} w_{i}\right)}{1-\langle u, w\rangle}\right| \leq \frac{2 r}{1-r} S_{w}=\frac{2 r}{1-r}\left(1-|w|^{2}\right)^{1 / 2} .
$$

Hence

$$
I_{2} \leq \frac{2 r}{1-r}\left(1-|w|^{2}\right)^{-1 / 2} \int_{E_{r}(w)}|\bar{R} f(z)| d \tau(z) .
$$

In (a) we have proved that the integral $I_{1}$ is also at most $C\left(1-|w|^{2}\right)^{-1 / 2} \times$ $\int_{E_{r}(w)}|\bar{R} f(z)| d \tau(z)$. This finishes the proof of Lemma 2.1.

Remark. In [12], p. 52, it is shown that $f(w)=\int_{S} f(z) h\left(\varphi_{z}(w)\right) d \tau(z)$, where $h$ is a radial function which belongs to $C^{\infty}(B)$ with compact support in $B$ such that $\int_{B} h(z) d \tau(z)=1$. Then the argument used in the proof of Lemma 2.1 can be applied to derive the estimate

$$
\left|T_{i j} f(w)\right| \leq C\left(1-|w|^{2}\right)^{-1 / 2} \int_{E_{r}(w)}|f(z)| d \tau(z), \quad w \in B, 1 \leq i, j \leq n .
$$

Proof of Theorem 1. In terms of ordinary differential operators the invariant Laplacian $\widetilde{\Delta}$ is as follows:

$$
\tilde{\Delta}=4\left(1-|z|^{2}\right) \sum_{j, k=1}^{n}\left(\delta_{j k}-z_{j} \overline{z_{k}}\right) \frac{\partial^{2}}{\partial z_{j} \partial \overline{z_{k}}},
$$

where $\delta_{j k}$ denotes the Kronecker delta; see [12], section 4.1, for details. Using this form for $\widetilde{\Delta}$ and the fact that $\widetilde{\Delta} f=\widetilde{\Delta} \bar{f}=0$ and $\overline{\partial f} / \partial z_{j}=\partial \bar{f} / \partial \overline{z_{j}}$, $1 \leq j \leq n$, we find that

$$
\tilde{\Delta}|f|^{2}(z)=2\left(1-|z|^{2}\right)\left|\nabla_{T} f(z)\right|^{2} .
$$

Also, $|\tilde{\nabla} f(z)|^{2}=2\left(|\widetilde{D} f(z)|^{2}+|\widetilde{D} \bar{f}(z)|^{2}\right)=\left(1-|z|^{2}\right)\left|\nabla_{T} f(z)\right|^{2}$ (see [12]). This proves the equivalences of (1), (2), and (3). 
An application of the Cauchy-Schwarz inequality shows that

$$
\left|\nabla_{T} f(z)\right|^{2} \geq 2\left(1-|z|^{2}\right)\left(|D f(z)|^{2}+|D \bar{f}(z)|^{2}\right)=\left(1-|z|^{2}\right)|\nabla f(z)|^{2} .
$$

Therefore, (3) implies (4). (We note that quantities $|\nabla f(z)|^{2}\left(1-|z|^{2}\right.$ ) and $\left|\nabla_{T} f(z)\right|^{2}$ are not pointwise equivalent if $n>1$. If $f$ is a function that depends on one variable only, say $z_{1}$, then it is not possible to bound $\left|\nabla_{T} f(z)\right|^{2}$ by $C\left(1-|z|^{2}\right)|\nabla f(z)|^{2}$ because $\left|\nabla_{T} f(z)\right|^{2}=\left(1-\left|z_{1}\right|^{2}\right)|\nabla f(z)|^{2}$.)

It is easy to see that (4) implies

$$
\sum_{j=1}^{n} \sup _{z \in B}\left(1-|z|^{2}\right)\left|\frac{\partial f}{\partial z_{j}}(z)\right|<\infty \text { and } \sum_{j=1}^{n} \sup _{z \in B}\left(1-|z|^{2}\right)\left|\frac{\partial f}{\partial \overline{z_{j}}}(z)\right|<\infty,
$$

which in turn implies

$$
\sup _{z \in B}\left(1-|z|^{2}\right)|R f(z)|<\infty \text { and } \sup _{z \in B}\left(1-|z|^{2}\right)|\bar{R} f(z)|<\infty .
$$

It is easy to check that

$$
|z|^{2}|D f(z)|^{2}=|R f(z)|^{2}+\sum_{i<j}\left|T_{i j} f(z)\right|^{2} .
$$

Using this, (2.1), and the definition of the tangential gradient we find that

$$
\begin{aligned}
|z|^{2} \tilde{\Delta}|f|^{2}(z)=4\left(1-|z|^{2}\right) & {\left[\left(1-|z|^{2}\right)\left(|R f(z)|^{2}+|R \bar{f}(z)|^{2}\right)\right.} \\
& \left.+\sum_{i<j}\left|T_{i j} f(z)\right|^{2}+\sum_{i<j}\left|T_{i j} \bar{f}(z)\right|^{2}\right] .
\end{aligned}
$$

Hence, by (2.1) and (2.2), to show that (5) implies (3) it is sufficient to show that

$$
\sum_{i<j} \sup _{z \in B}\left(1-|z|^{2}\right)^{1 / 2}\left[\left|T_{i j} f(z)\right|+\left|\bar{T}_{i j} f(z)\right|\right]<\infty .
$$

An integration by parts shows that

$$
f(z)=\int_{0}^{1}[R f(t z)+\bar{R} f(t z)+f(t z)] d t .
$$

From this we conclude that it is sufficient to prove that

$$
\int_{0}^{1}\left|T_{i j} u(t z)\right| d t=O\left(\frac{1}{\sqrt{1-|z|^{2}}}\right), \quad 1 \leq i<j \leq n,
$$

where $u(z)=R f(z)$ or $\bar{R} f(z)$ or $R \bar{f}(z)$ or $\bar{R} \bar{f}(z)$ or $f(z)$.

From

$$
f(z)-f(0)=\int_{0}^{1} \frac{d}{d t} f(t z) d t=\int_{0}^{1} \frac{1}{t}(R f(t z)+\bar{R} f(t z)) d t, \quad z \in B,
$$

we see that $f(z)=O\left(\frac{1}{1-|z|}\right)$ (in fact, $f(z)=O\left(\log \frac{1}{1-|z|}\right)$ ). Thus, $u(z)=$ $O\left(\frac{1}{1-|z|}\right)$ (note that if $f \in \mathscr{M}$, then $\bar{f} \in \mathscr{M}$ and $|\nabla f(z)|=|\nabla \bar{f}(z)|, z \in B$ ). Using this, Lemma 2.1 , the estimate obtained in the remark following Lemma 
2.1 , the fact that $1-|w|^{2} \cong 1-|z|^{2}$, for $w \in E_{r}(z)$, and the invariance of measure $\tau$ we find that

$$
\begin{aligned}
\int_{0}^{1}\left|T_{i j} u(t z)\right| d t & \leq C \int_{0}^{1}\left[\frac{1}{(1-t|z|)^{1 / 2}} \int_{E_{r}(t z)}|u(w)| d \tau(w)\right] d t \\
& \leq C \int_{0}^{1} \frac{d t}{(1-t|z|)^{3 / 2}} \leq \frac{C}{(1-|z|)^{1 / 2}} .
\end{aligned}
$$

\section{Proof of Theorem 3}

Lemma 3.1. Let $k \geq m$ be positive integers, $0<p<\infty$, and $0<r<1$. There exists a constant $C=C(k, m, p, r, n)$ such that if $f \in \mathscr{M}$, then

$$
\left|\partial^{k} f(w)\right|^{p} \leq C(1-|w|)^{(m-k) p} \int_{E_{r}(w)}\left|\partial^{m} f(z)\right|^{p} d \tau(z), \quad \text { for all } w \in B .
$$

Proof. Let $\alpha$ and $\beta$ be multi-indices. Using the formula (1.3) in [1] again we find that

$$
\begin{aligned}
& F\left(-|\beta|,-|\alpha|, n ; r^{2}\right) \partial^{\alpha} \bar{\partial}^{\beta} f(w) \\
& \quad=\int_{S}(1-\langle w, r \xi\rangle)^{-|\alpha|}(1-\langle r \xi, w\rangle)^{-|\beta|} \partial^{\alpha} \bar{\partial}^{\beta} f(r \xi) d \sigma(\xi),
\end{aligned}
$$

where $f(a, b, c ; x)$ denotes the usual hypergeometric function. Multiplying this equality by $2 n r^{2 n-1}\left(1-r^{2}\right)^{-n-1} h(r) d r$, where $h$ is a radial function which belongs to $C^{\infty}(B)$ with compact support in $B$ such that

$$
\int_{B} F\left(-|\beta|,-|\alpha|, n ;|z|^{2}\right) h(z) d \tau(z)=1
$$

and then integrating from 0 to 1 and using the invariance of the measure $\tau$, we get

$$
\begin{aligned}
\partial^{\alpha} \bar{\partial}^{\beta} f(w) & =\int_{B} h\left(\varphi_{w}(z)\right) \frac{\partial^{\alpha} \bar{\partial}^{\beta} f(z) d \tau(z)}{\left(1-\left\langle w, \varphi_{w}(z)\right\rangle\right)^{|\alpha|}\left(1-\left\langle\varphi_{w}(z), w\right\rangle\right)^{|\beta|}} \\
& =\int_{B} h\left(\varphi_{w}(z)\right) \frac{(1-\langle w, z\rangle)^{|\alpha|}(1-\langle z, w\rangle)^{|\beta|}}{\left(1-|w|^{2}\right)^{|\alpha|+|\beta|}} \partial^{\alpha} \bar{\partial}^{\beta} f(z) d \tau(z),
\end{aligned}
$$

by Theorem 2.2.2 ([12], p. 26).

Since

$$
|1-\langle z, w\rangle| \cong 1-|w|^{2}, \quad z \in E_{r}(w),
$$

by a suitable choice of a function $h$ we obtain

$$
\left|\partial^{\alpha} \bar{\partial}^{\beta} f(w)\right| \leq C \int_{E_{r}(w)}\left|\partial^{\alpha} \bar{\partial}^{\beta} f(z)\right| d \tau(z) .
$$

Hence,

$$
\left|\partial^{m} f(w)\right| \leq C \int_{E_{r}(w)}\left|\partial^{m} f(z)\right| d \tau(z) .
$$

By Lemma 2.4 ([11]) (see also [2]) we find that

$$
\left|\partial^{m} f(w)\right|^{p} \leq C \int_{E_{r}(w)}\left|\partial^{m} f(z)\right|^{p} d \tau(z) .
$$


By differentiating under the integral sign in (3.1), using the formula for $\varphi_{z}(w)$ ([12]), and arguing as above we conclude that

$$
\left|D_{j} \partial^{\alpha} \bar{\partial}^{\beta} f(w)\right| \leq \frac{C}{1-|w|} \int_{E_{r}(w)}\left|\partial^{\alpha} \bar{\partial}^{\beta} f(z)\right| d \tau(z), \quad w \in B, 1 \leq j \leq n,
$$

and

$$
\left|\bar{D}_{j} \partial^{\alpha} \bar{\partial}^{\beta} f(w)\right| \leq \frac{C}{1-|w|} \int_{E_{r}(w)}\left|\partial^{\alpha} \bar{\partial}^{\beta} f(z)\right| d \tau(z), \quad w \in B, \quad 1 \leq j \leq n,
$$

and so,

$$
\left|\partial^{m+1} f(w)\right| \leq \frac{C}{1-|w|} \int_{E_{r}(w)}\left|\partial^{m} f(z)\right| d \tau(z) .
$$

By an adaptation of the argument given in ([11], Lemma 2.4) we find that

$$
\left|\partial^{m+1} f(w)\right|^{p} \leq \frac{C}{(1-|w|)^{p}} \int_{E_{r}(w)}\left|\partial^{m} f(z)\right|^{p} d \tau(z) .
$$

An induction argument shows that

$$
\left|\partial^{k} f(w)\right|^{p} \leq \frac{C}{(1-|w|)^{(k-m) p}} \int_{E_{r}(w)}\left|\partial^{m} f(z)\right|^{p} d \tau(z) .
$$

Proof of Theorem 3. The equivalence of (i) and (ii) is proved in Theorem 1.

If $z \in E_{r}(w)$, then $1-|w|^{2} \cong 1-|z|^{2}$. Hence by Lemma 3.1

$$
(1-|z|)^{m}\left|\partial^{m} f(z)\right| \leq C \int_{E_{r}(z)}(1-|w|)|\partial f(w)| d \tau(w) \leq C\|f\|_{\mathscr{B}} \tau\left(E_{r}(z)\right),
$$

by Theorem 1. Since $\tau\left(E_{r}(z)\right)=r^{2 n}\left(1-r^{2}\right)^{-n}$, we have that (ii) $\Rightarrow$ (iii).

Conversely, assuming that $\partial^{\alpha} \bar{\partial}^{\beta} f(0)=0$ we have

$$
\left|\partial^{\alpha} \bar{\partial}^{\beta} f(z)\right| \leq \int_{0}^{1}\left|\frac{d}{d r} \partial^{\alpha} \bar{\partial}^{\beta} f(r z) d r\right| \leq C \int_{0}^{1}\left|\partial^{|\alpha|+|\beta|+1} f(r z)\right| d r .
$$

Hence,

$$
\left|\partial^{k} f(z)\right| \leq C \int_{0}^{1}\left|\partial^{k+1} f(t z)\right| d t,
$$

for any positive integer $k$. The implication (iii) $\Rightarrow$ (ii) follows at once.

Since $\tau\left(E_{r}(w)\right)$ is bounded by a constant independent of $w$, we have that (iii) $\Rightarrow$ (iv).

Let $k \geq m$ be a positive integer. Then by Lemma 3.1 we have

$$
(1-|z|)^{k p}\left|\partial^{k} f(z)\right|^{p} \leq C \int_{E_{r}(z)}\left|\partial^{m} f(w)\right|^{p}(1-|w|)^{m p} d \tau(w) .
$$

Thus, (iv) implies that $\sup _{z \in B}(1-|z|)^{k}\left|\partial^{k} f(z)\right|<\infty$.

This finishes the proof of Theorem 3 .

\section{ACKNOWLEDGMENT}

We wish to thank the referee for carefully reading the manuscript and making several useful suggestions for improvement (and in some instances corrections) of the paper. In particular, the referee significantly simplified the proof of the equality (2.2) and part of the proof of Lemma 2.1. 


\section{REFERENCES}

1. P. Ahern and C. Cascante, Exceptional sets for Poisson integral of potentials on the unit sphere in $C^{n}, p \leq 1$, Pacific J. Math. 153 (1992), 1-15.

2. P. Ahern and J. Bruna, Maximal and area integral characterization of Hardy-Sobolev spaces in the unit ball of $C^{n}$, Rev. Mat. Iberoamericana 4 (1988), 123-153.

3. J. M. Anderson, J. G. Clunie, and C. H. Pommerenki, On Bloch functions and normal functions, J. Reine Angew. Math. 270 (1974), 12-37.

4. J. Arazy, S. Fisher, S. Janson, and J. Peetre, Membership of Hankel operators on the ball in unitary ideals, J. London Math. Soc. 43 (1991), 485-508.

5. S. Axler, The Bergman space, the Bloch space and commutators of multiplication operators, Duke Math. J. 53 (1986), 315-332.

6. F. Beatrous and J. Burbea, Holomorphic Sobolev spaces on the ball, Dissertationes Math. 256 (1989), 1-57.

7. D. Geller, Some results on $H^{p}$ theory for the Heisenberg group, Duke Math. J. 47 (1980), 365-390.

8. K. Hahn and E. Youssfi, Möbius invariant Besov p-spaces and Hankel operators in the Bergman space on the ball in $C^{n}$, Complex Variables 17 (1991), 89-104.

9. __ Manuscripta Math. 71 (1991), 67-81.

10. M. Jevtic, On the Carleson measure characterization of BMO functions on the unit sphere, Proc. Amer. Math. Soc. (to appear).

11. M. Pavlovic, Inequalities for the gradient of eigenfunctions of the invariant Laplacian in the unit ball, Indag. Math. 2 (1991), 89-98.

12. W. Rudin, Function theory in the unit ball of $C^{n}$, Springer-Verlag, New York, 1980.

13. K. Stroethoff, Besov-type characterizations for the Bloch space, Bull. Austral. Math. Soc. 39 (1989), 405-420.

14. R. Timoney, Bloch functions in several complex variables. I, Bull. London Math. Soc. 12 (1980), 241-267.

Matematički Fakultet, Studentski TRg 16, 11000 Beograd, Yugoslavia 